\title{
FILTRATIONS IN SEMISIMPLE LIE ALGEBRAS, II
}

\author{
Y. BARNEA AND D. S. PASSMAN
}

\begin{abstract}
In this paper, we continue our study of the maximal bounded $\mathbb{Z}$-filtrations of a complex semisimple Lie algebra $L$. Specifically, we discuss the functionals which give rise to such filtrations, and we show that they are related to certain semisimple subalgebras of $L$ of full rank. In this way, we determine the "order" of these functionals and count them without the aid of computer computations. The main results here involve the Lie algebras of type $E_{6}, E_{7}$ and $E_{8}$, since we already know a good deal about the functionals for the remaining types. Nevertheless, we reinterpret our previous results into the new context considered here. Finally, we describe the associated graded Lie algebras of all of the maximal filtrations obtained in this manner.
\end{abstract}

\section{INTRODUCTION}

Let $L$ be a Lie algebra over a field $K$. A $\mathbb{Z}$-filtration $\mathcal{F}=\left\{F_{i} \mid i \in \mathbb{Z}\right\}$ of $L$ is a collection of $K$-subspaces

$$
\cdots \subseteq F_{-2} \subseteq F_{-1} \subseteq F_{0} \subseteq F_{1} \subseteq F_{2} \subseteq \cdots
$$

indexed by the integers $\mathbb{Z}$ such that $\left[F_{i}, F_{j}\right] \subseteq F_{i+j}$ for all $i, j \in \mathbb{Z}$. One usually also assumes that $\bigcup_{i} F_{i}=L$ and $\bigcap_{i} F_{i}=0$. In particular, $F_{0}$ is a Lie subalgebra of $L$ and each $F_{i}$ is an $F_{0}$-Lie submodule of $L$. Furthermore, we say that the filtration is bounded if there exist integers $\ell$ and $\ell^{\prime}$ with $F_{\ell}=0$ and $F_{\ell^{\prime}}=L$. In this case, it is clear that each $F_{i}$, with $i<0$, is ad-nilpotent in its action on $L$.

If $\mathcal{G}=\left\{G_{i} \mid i \in \mathbb{Z}\right\}$ is a second such filtration, we say that $\mathcal{G}$ contains $\mathcal{F}$, or $\mathcal{G}$ is larger than $\mathcal{F}$, if $G_{i} \supseteq F_{i}$ for all $i$. In particular, it makes sense to speak about maximal bounded filtrations and, in case $L$ is a complex semisimple Lie algebra, such filtrations are essentially classified in [BP]. Indeed, by [BP, Lemma 1.4(ii)], the classification problem reduces immediately to the case of finite-dimensional simple Lie algebras over $\mathbb{C}$. Thus $L$ is either one of the classical algebras of type $A_{n}, B_{n}$, $C_{n}, D_{n}$, or one of the five exceptional algebras $E_{6}, E_{7}, E_{8}, F_{4}, G_{2}$.

While the results of $[\mathrm{BP}]$ are complete for the four classical infinite series, some questions remain for the five exceptional algebras. In this paper, we answer one of these questions in a reasonably noncomputational manner. Specifically, we describe the linear functionals $\lambda: V \rightarrow \mathbb{R}$ that determine maximal filtrations $\mathcal{F}_{\lambda}$. As will be apparent, the filtrations themselves play almost no role here; basically, we study root systems.

Received by the editors August 10, 2004 and, in revised form, October 19, 2005.

2000 Mathematics Subject Classification. Primary 17B20, 17B70, 16W70.

The first author's research was carried out while visiting the departments of Mathematics at Imperial College and at the University of Kent. He thanks both departments.

The second author's research was supported in part by NSA grant 144-LQ65. 
We start by setting notation. To this end, let $L$ be a simple finite-dimensional Lie algebra over $\mathbb{C}$, denote by $L_{0}$ a fixed Cartan subalgebra, and let

$$
L=L_{0}+\sum_{\alpha \in \Phi} L_{\alpha}
$$

describe the root space decomposition of $L$. Thus $\Phi$ is the set of roots of $L$, and we know that each root space $L_{\alpha}$, with $\alpha \in \Phi$, is one-dimensional. Furthermore, $\Phi$ is contained naturally in a real inner product space $V$ and it spans that space. Indeed, $n=\operatorname{dim}_{\mathbb{R}} V=\operatorname{dim}_{\mathbb{C}} L_{0}$ is the rank of $L$.

For convenience, set $\Phi^{\prime}=\Phi \cup\{0\}$. If $\lambda: V \rightarrow \mathbb{R}$ is a linear functional, then $\lambda$ determines a $\mathbb{Z}$-filtration $\mathcal{F}_{\lambda}=\left\{F_{i} \mid i \in \mathbb{Z}\right\}$ by defining $F_{i}=\sum L_{\alpha}$, where the sum is over all $\alpha \in \Phi^{\prime}$ with $\lambda(\alpha) \leq i$. Certainly, each such $\mathcal{F}_{\lambda}$ is bounded and the problem is to describe, in a fairly precise manner, the set of all functionals $\lambda$ such that $\mathcal{F}_{\lambda}$ is maximal. One characterization, in [BP, Proposition 4.3], is given by

Lemma 1.1. The filtration $\mathcal{F}_{\lambda}$ is maximal if and only if

$$
\Phi_{\lambda}=\{\alpha \in \Phi \mid \lambda(\alpha) \in \mathbb{Z}\}
$$

spans the vector space $V$.

We say that such functionals $\lambda$ are maximal, and we use $\mathfrak{M}$ to denote the subset of $\widehat{V}=\operatorname{Hom}_{\mathbb{R}}(V, \mathbb{R})$ consisting of all maximal $\lambda$. Note that rigid might be a more appropriate name for these functionals since they are anchored by the integer values they take on. Indeed, any perturbation of $\lambda$, no matter how small, will necessarily move some $\lambda(\alpha)$, with $\alpha \in \Phi_{\lambda}$, from its present integer value and hence change the position of at least one of $L_{\alpha}$ or $L_{-\alpha}$ in the filtration $\mathcal{F}_{\lambda}$. Furthermore, it is easy to see from the above that this rigidity property characterizes the functionals in $\mathfrak{M}$.

Again, by the preceding lemma, if

$$
\Lambda_{\Phi}=\{\lambda \in \widehat{V} \mid \lambda(\Phi) \subseteq \mathbb{Z}\}
$$

then $\Lambda_{\Phi}$ is a subgroup of $\widehat{V}$, clearly isomorphic to $\mathbb{Z}^{n}$, and with $\Lambda_{\Phi} \subseteq \mathfrak{M}$. In addition, by [BP, Corollary 4.4(iii)], $\mathfrak{M}$ is a finite union of cosets of $\Lambda_{\Phi}$, and the goal here is to better understand $\mathfrak{M}$, to determine the orders of the elements of $\mathfrak{M}$ modulo $\Lambda_{\Phi}$, and to count the number of $\Lambda_{\Phi}$-cosets in $\mathfrak{M}$. For this, we define

$$
P_{\lambda}=L_{0}+\sum_{\alpha \in \Phi_{\lambda}} L_{\alpha}
$$

for any $\lambda \in \mathfrak{M}$, and standard arguments now yield

Lemma 1.2. If $\lambda$ is maximal, then

i. $\lambda(\Phi) \subseteq \mathbb{Q}$.

ii. $P_{\lambda}$ is a semisimple Lie subalgebra of $L$ with Cartan subalgebra $L_{0}$, root system $\Phi_{\lambda}$, and full rank $n$.

Proof. (i) Let $\Sigma$ be a set of simple roots for $L$. Then $\Sigma$ is a basis for $V$, and indeed each root in $\Phi$ is an integral linear combination of the members of $\Sigma$. In particular, since $\Phi_{\lambda}$ spans $V$, it follows, by inverting an integer matrix, that the members of $\Sigma$ are rational linear combinations of the members of $\Phi_{\lambda}$. Hence $\lambda(\Sigma) \subseteq \mathbb{Q}$, and then $\lambda(\Phi) \subseteq \mathbb{Q}$. Of course, this fact also follows from [BP, Lemma 3.6].

(ii) It is clear that $\Phi_{\lambda}$ is closed under negatives and sums, where the latter means that if $\alpha, \beta \in \Phi_{\lambda}$ and $\alpha+\beta \in \Phi$, then $\alpha+\beta \in \Phi_{\lambda}$. Thus $P_{\lambda}$ is certainly a Lie 
subalgebra of $L$ containing $L_{0}$. Next, note that $L_{0}$ is abelian and self-normalizing in $L$, so it is abelian and self-normalizing in $P_{\lambda}$. Thus $L_{0}$ is a Cartan subalgebra of $P_{\lambda}$ and $\Phi_{\lambda}$ is clearly its root system. It remains to show that $P_{\lambda}$ is semisimple. To this end, let $A$ be an abelian ideal of the Lie algebra. Then $L_{0} \cap A$ is nilpotent in its ad-action on $P_{\lambda}$ and, of course, it is semisimple in its action on $L$. It follows that $L_{0} \cap A$ is central in $P_{\lambda}$ and, in particular, $\alpha\left(L_{0} \cap A\right)=0$ for all $\alpha \in \Phi_{\lambda}$. But $\Phi_{\lambda}$ spans $V$, so $L_{0} \cap A \subseteq L_{0}$ is trivial on all the roots in $\Phi$, and hence $L_{0} \cap A=0$. Finally, if $A \neq 0$, then, since $A$ is an ad $L_{0}$-submodule of $P_{\lambda}$ and since $L_{0} \cap A=0$, we must have $L_{\alpha} \subseteq A$ for some $\alpha \in \Phi_{\lambda}$. But then $-\alpha \in \Phi_{\lambda}$, so $0 \neq\left[L_{\alpha}, L_{-\alpha}\right] \subseteq A$, and hence $0 \neq L_{0} \cap A$, a contradiction.

If $\lambda$ and $\mu$ are maximal functionals in the same coset of $\Lambda_{\Phi}$, then it is clear that $\Phi_{\lambda}=\Phi_{\mu}$ and hence that the corresponding $P_{\lambda}$ and $P_{\mu}$ are equal. Furthermore, as we will see in the remainder of this paper, the structure of the Lie subalgebra $P_{\lambda}$ contains all the ingredients necessary to understand the functional $\lambda$ modulo $\Lambda_{\Phi}$. Indeed, for $\lambda \in \mathfrak{M} \backslash \Lambda_{\Phi}$, we will show:

- $P_{\lambda}$ is a one-step subalgebra of $L$. By this, we mean that $P_{\lambda}$ has a set $\Sigma_{\lambda}$ of simple roots which can be obtained from a completed Dynkin diagram $\bar{\Sigma}$ of $L$ by deleting a single node. In other words, $\Sigma=\left\{\alpha_{1}, \alpha_{2}, \ldots, \alpha_{n}\right\}$ is a set of simple roots of $L, \bar{\Sigma}$ the extension of $\Sigma$ obtained by adjoining the lowest root $-\delta$, and $\Sigma_{\lambda}=\bar{\Sigma} \backslash\left\{\alpha_{k}\right\}$ for some $k$.

- If $\delta=c_{1} \alpha_{1}+c_{2} \alpha_{2}+\cdots+c_{n} \alpha_{n}$ is the corresponding highest root of $L$, with $c_{i} \in \mathbb{Z}$, and if $\Sigma_{\lambda}=\bar{\Sigma} \backslash\left\{\alpha_{k}\right\}$, then $c=c_{k}$ is the order of $\lambda$ modulo $\Lambda_{\Phi}$. In particular, the possibilities for $c$ are $2,3,4,5$ or 6 , depending of course on the isomorphism type of $L$.

- Again, if $c=c_{k}$ is the order of $\lambda$ modulo $\Lambda_{\Phi}$, then there is a natural one-toone correspondence between the subgroups of $\mathbb{Z} / c \mathbb{Z} \cong(\lambda(\Phi)+\mathbb{Z}) / \mathbb{Z}$ and the semisimple subalgebras of $L$ containing $P_{\lambda}$. In particular, $P_{\lambda}$ is maximal if and only if $c=2,3$ or 5 is prime.

- Let $\mathcal{W}(\Phi)$ denote the Weyl group of the set $\Phi$ of roots of $L$. Then the number of one-step subalgebras $P$ of $L$ containing $L_{0}$ and isomorphic to $P_{\lambda}$ is equal to $|\mathcal{W}(\Phi)| /\left|\mathcal{W}\left(\Phi_{\lambda}\right)\right|$ divided by a small integer which we call the index. The index is of size 1,2, 4 or 6 and depends upon the geometry of $\Sigma$ and of $\Sigma_{\lambda}$.

- The number of cosets $\left(\Lambda_{\Phi}\right) \mu \subseteq \mathfrak{M}$ with $P_{\mu} \cong P_{\lambda}$ is equal to the product of the number of $P \cong P_{\lambda}$, as given above, and $\phi(c)$, where $\phi$ is the Euler function and $c$ is the order of $\lambda$ modulo $\Lambda_{\Phi}$.

- If $G_{\lambda}$ denotes the associated graded Lie algebra of the filtration $\mathcal{F}_{\lambda}$, then $G_{\lambda}=N_{\lambda} \rtimes P_{\lambda}$, where $N_{\lambda}=\operatorname{rad} G_{\lambda}$ is nilpotent of class $<c$. Furthermore, $N_{\lambda}$ is a $\mathbb{Z} / c \mathbb{Z}$-graded Lie algebra, it has trivial 0 -component, and it is isomorphic to $L / P_{\lambda}$ as an ad $P_{\lambda}$-module, with each nonzero component an irreducible ad $P_{\lambda}$-submodule.

Conversely, any one-step subalgebra of $L$ containing the Cartan subalgebra $L_{0}$ is a suitable $P_{\lambda}$ with $\lambda \in \mathfrak{M} \backslash \Lambda_{\Phi}$.

In view of Lemmas 1.1 and 1.2(i), we introduce a measure of the distance between a rational number $q$ and $\mathbb{Z}$. To this end, let $\kappa$ denote the natural homomorphism $\kappa: \mathbb{Q} \rightarrow \mathbb{Q} / \mathbb{Z}$ onto the torsion group $\mathbb{Q} / \mathbb{Z}$. Then $\kappa(q)$ has finite order, and we call this number the order of $q$. Note that, if $q=a / b$ with $a$ and $b$ relatively prime 
integers and with $b>0$, then the order of $q$ is precisely equal to $b$. Similarly, if $\lambda: V \rightarrow \mathbb{R}$ is a functional with $\lambda(\Phi) \subseteq \mathbb{Q}$, then the order of $\lambda$ is the smallest positive integer $c$ such that $c \cdot \lambda(\Phi) \subseteq \mathbb{Z}$. In particular, the order of $\lambda$ is the least common multiple of the orders of the various $\lambda(\alpha)$ with $\alpha \in \Phi$. Note that $\kappa \circ \lambda=\kappa \circ \mu$ if and only if $\lambda$ and $\mu$ determine the same coset modulo $\Lambda_{\Phi}$, and the order of $\lambda$ is precisely its group-theoretic order modulo $\Lambda_{\Phi}$.

Our study of the semisimple subalgebras $P_{\lambda}$ of $L$ uses the techniques and results of [D, Chapter 2]. Since that paper has a number of typographical errors, we will take care when quoting its results.

\section{MAXIMAL FUNCTIONALS}

Let $\lambda$ be a maximal (or rigid) functional on $V$ not contained in $\Lambda_{\Phi}$. Our goal here is to study its corresponding semisimple subalgebra $P_{\lambda}$. As in [D], we are concerned with certain subsets $\Gamma$ of the root set $\Phi$ that satisfy

$$
\text { if } \alpha, \beta \in \Gamma \text {, then } \alpha-\beta \notin \Phi \text {. }
$$

In particular, any set of simple roots satisfies $(*)$. Now let $\Sigma_{\lambda}$ denote a set of simple roots for $P_{\lambda}$. Then we have

Lemma 2.1. If $\alpha \in \Phi \backslash \Phi_{\lambda}$, then there exists a root $\alpha^{\prime} \in \Phi$ such that

$i$. $\alpha^{\prime}$ is obtained from $\alpha$ by successively subtracting elements of $\Sigma_{\lambda}$.

ii. $\alpha$ is obtained from $\alpha^{\prime}$ by successively adding elements of $\Sigma_{\lambda}$.

iii. $\lambda\left(\alpha^{\prime}\right) \equiv \lambda(\alpha) \bmod \mathbb{Z}$, so $\alpha^{\prime} \in \Phi \backslash \Phi_{\lambda}$.

iv. $\Sigma_{\lambda} \cup\left\{\alpha^{\prime}\right\}$ satisfies condition $(*)$.

Proof. We construct a sequence of roots $\alpha_{0}, \alpha_{1}, \alpha_{2}, \ldots$ of $L$, with $\alpha_{0}=\alpha$, as follows. Assume $\alpha_{i} \in \Phi$ is given. If there exists $\sigma_{i} \in \Sigma_{\lambda}$ with $\alpha_{i}-\sigma_{i} \in \Phi$, then set $\alpha_{i+1}=\alpha_{i}-\sigma_{i}$. Since the roots in $\Sigma_{\lambda}$ are linearly independent, it is clear that the members of the $\alpha$-sequence are distinct and hence this procedure must terminate in a finite number of steps. If it terminates at $i=j$, then we set $\alpha^{\prime}=\alpha_{j}$.

Let us fix one choice of $\alpha^{\prime}$ for each root $\alpha \in \Phi \backslash \Phi_{\lambda}$. If $\widetilde{M}$ is a simple subalgebra of $L$ and if $\left\{\alpha_{1}, \alpha_{2}, \ldots, \alpha_{r}\right\}$ is a set of simple roots of $\widetilde{M}$, then the highest root $\delta$ can be written as a positive integer linear combination $\delta=c_{1} \alpha_{1}+c_{2} \alpha_{2}+\cdots+c_{r} \alpha_{r}$ where the $c_{i}$ s are the coefficients that occur in the following statement.

Lemma 2.2. If $\alpha \in \Phi \backslash \Phi_{\lambda}$, then there exists a semisimple subalgebra $M$ of $L$ of full rank such that

i. $M \supseteq P_{\lambda}$ and $M \supseteq L_{\alpha}$.

ii. The order of $\lambda(\alpha)$ divides one of the coefficients of a highest root in a simple direct summand $\widetilde{M}$ of $M$.

iii. If $\lambda(\alpha)$ has order $\geq 3$, then $P_{\lambda}$ is not isomorphic to $n A_{1}$.

In particular, if $P_{\lambda}$ and $L_{\alpha}$ generate $L$, then there exists a set $\Sigma=\left\{\alpha_{1}, \alpha_{2}, \ldots, \alpha_{n}\right\}$ of simple roots of $\Phi$, with highest root $\delta=c_{1} \alpha_{1}+c_{2} \alpha_{2}+\cdots+c_{n} \alpha_{n}$, such that, for some subscript $j$, the order of $\lambda(\alpha)$ divides $c_{j}$, and $\Sigma_{\lambda}$ consists of $(-\delta)$ and those simple roots $\alpha_{i}$ with $i \neq j$.

Proof. By the previous lemma, we know that $\Sigma_{\lambda} \cup\left\{\alpha^{\prime}\right\}$ satisfies (*). Furthermore, this set has size $n+1=\operatorname{rank} L+1$. Thus, by [D, Section 5] (see also [K, Chapter 4]), we can write

$$
\Sigma_{\lambda} \cup\left\{\alpha^{\prime}\right\}=\bar{\Delta}_{1} \cup \Delta_{2} \cup \cdots \cup \Delta_{k}
$$


where the $\Delta_{i}$ are mutually orthogonal and where each $\Delta_{i}$ is the set of simple roots of a simple Lie algebra. Furthermore, $\bar{\Delta}_{1}$ is the extension of $\Delta_{1}$ obtained by adjoining the lowest root of the root system it spans. In particular, if $\Delta=\Delta_{1} \cup \Delta_{2} \cup \cdots \cup \Delta_{k}$ and if $M$ is the Lie subalgebra of $L$ generated by $L_{0}$ and the various $L_{\beta}$ with $\beta \in \pm \Delta$, then $M$ is a semisimple subalgebra of $L$ of full rank containing $P_{\lambda}$ and $L_{\alpha^{\prime}}$. Thus it also contains $L_{\alpha}$. Let $\widetilde{M}$ be the simple direct summand of $M$ corresponding to the simple root set $\Delta_{1}$.

Next, we consider which position the root $\alpha^{\prime}$ occupies in the union $\Sigma_{\lambda} \cup\left\{\alpha^{\prime}\right\}=$ $\bar{\Delta}_{1} \cup \Delta_{2} \cup \cdots \cup \Delta_{k}$. To start with, it must be contained in $\bar{\Delta}_{1}$, since otherwise deleting $\alpha^{\prime}$ would lead to a linearly dependent set of roots. Next, $\alpha^{\prime}$ cannot correspond to the lowest root in $\bar{\Delta}_{1}$, since otherwise $\alpha^{\prime}$ would be contained in $\Phi_{\lambda}$. Thus, if $\Delta_{1}=$ $\left\{\gamma_{1}, \gamma_{2}, \ldots, \gamma_{r}\right\}$ and if the highest root $\delta$ is written as $\delta=c_{1} \gamma_{1}+c_{2} \gamma_{2}+\cdots+c_{r} \gamma_{r}$, then $\alpha^{\prime}=\gamma_{j}$ for some $j$, while the remaining $\gamma_{i}$, along with $-\delta$, are contained in $\Sigma_{\lambda}$. In particular, by applying $\lambda$ to the above linear relation, we see that $c_{j} \lambda\left(\alpha^{\prime}\right)=$ $c_{j} \lambda\left(\gamma_{j}\right) \in \mathbb{Z}$ and hence $c_{j} \lambda(\alpha) \in \mathbb{Z}$, as required.

If $\lambda(\alpha)$ has order $\geq 3$, then $c_{j} \geq 3$ and hence $\Delta_{1}$ must be the set of simple roots for one of the five exceptional simple Lie algebras. It is now easy to determine, from [Bo, Plates V-IX], all the isomorphism classes obtained by deleting a node from $\bar{\Delta}_{1}$ corresponding to a coefficient $\geq 3$, and none of these is isomorphic to the standard Dynkin diagram for the direct sum of copies of $A_{1}$. Finally, if $P_{\lambda}$ and $L_{\alpha}$ generate $L$, then (i) implies that $M=L$. Thus, since $L$ is simple, we must have $\widetilde{M}=L$ and $\Delta=\Delta_{1}$. With this, the result is clear.

As an immediate consequence of the above, along with [Bo, Plates I-IX] and [D, Theorem 5.3], we obtain

Lemma 2.3. Let $\lambda: V \rightarrow \mathbb{Q}$ be a maximal functional. If $\alpha \in \Phi$, then the possible orders for $\lambda(\alpha)$ are given in the following table.

\begin{tabular}{||l|l||}
\hline \hline Type & Order \\
\hline \hline$A_{n}$ & 1 \\
$B_{n}, C_{n}, D_{n}$ & 1,2 \\
$E_{6}$ & $1,2,3$ \\
$E_{7}$ & $1,2,3,4$ \\
$E_{8}$ & $1,2,3,4,5,6$ \\
$F_{4}$ & $1,2,3,4$ \\
$G_{2}$ & $1,2,3$ \\
\hline \hline
\end{tabular}

In particular, we see that $\lambda$ has order 1 if $L=A_{n}$, and that $\lambda$ has order 1 or 2 if $L$ is of type $B_{n}, C_{n}$ or $D_{n}$.

Recall that a proper semisimple subalgebra $P$, containing $L_{0}$, is said to be a onestep subalgebra if there exists a set $\Delta$ of simple roots of $P$ which can be obtained from $\bar{\Sigma}$, a completed diagram of $L$, by deleting a node.

Lemma 2.4. Let $\lambda: V \rightarrow \mathbb{R}$ be a maximal functional of order $\neq 1$, and let $\alpha$ and $\beta$ be roots in $\Phi \backslash \Phi_{\lambda}$ with $\alpha^{\prime} \neq \beta^{\prime}$. If either $L$ is classical and $P_{\lambda}$ is isomorphic to a one-step subalgebra, or $L$ is a simple exceptional Lie algebra, then $\alpha^{\prime}-\beta^{\prime} \in \Phi$. 
Proof. If $\alpha^{\prime}-\beta^{\prime}$ is not a root, then Lemma 2.1 implies that $\Sigma_{\lambda} \cup\left\{\alpha^{\prime}, \beta^{\prime}\right\}$ satisfies $(*)$, and $[\mathrm{D}$, Section 5] yields

$$
\Sigma_{\lambda} \cup\left\{\alpha^{\prime}, \beta^{\prime}\right\}=\bar{\Delta}_{1} \cup \bar{\Delta}_{2} \cup \Delta_{3} \cup \cdots \cup \Delta_{k},
$$

where each $\Delta_{i}$ is a set of simple roots for a simple Lie subalgebra of $L$, where the $\Delta_{i}$ are mutually orthogonal, and where $\bar{\Delta}_{1}$ and $\bar{\Delta}_{2}$ are the extensions of $\Delta_{1}$ and $\Delta_{2}$, respectively, obtained by adjoining the lowest root of the root system each one spans. As in the proof of Lemma 2.2, we see that $\alpha^{\prime} \in \bar{\Delta}_{1}, \beta^{\prime} \in \bar{\Delta}_{2}$ and that these elements must correspond to simple roots with coefficients $\geq 2$.

Suppose first that $L$ is of classical type and that $P_{\lambda}$ is isomorphic to a one-step subalgebra. Since the coefficients above are $\geq 2$, we have $L ¥ A_{n}$. If both $\bar{\Delta}_{1} \backslash\left\{\alpha^{\prime}\right\}$ and $\bar{\Delta}_{2} \backslash\left\{\beta^{\prime}\right\}$ are disconnected, then $\Sigma_{\lambda}$ has at least four connected components, a contradiction since $P_{\lambda}$ is isomorphic to a one-step subalgebra of $L$. Thus we can suppose that $\bar{\Delta}_{1} \backslash\left\{\alpha^{\prime}\right\}$ is connected. This implies that $\Delta_{1}$ corresponds to $B_{k}$ and that $\bar{\Delta}_{1} \backslash\left\{\alpha^{\prime}\right\}$ corresponds to $D_{k}$ with $k \geq 3$. In particular, the roots in $\Phi$ have two different lengths, so $L$ is of type $B_{n}$ or $C_{n}$. Furthermore, since $\Sigma_{\lambda}$ has a summand isomorphic to $D_{k}$ with $k \geq 3$, it follows that $L$ has type $B_{n}$ and $P_{\lambda} \cong D_{k}+B_{n-k}$. But then $\Sigma_{\lambda}$ has only two connected components, so $\bar{\Delta}_{2} \backslash\left\{\beta^{\prime}\right\}$ is also connected and hence isomorphic to some $D_{\ell}$ with $\ell \geq 3$, a contradiction.

Now let $L$ be an exceptional simple Lie algebra. Again, using the fact that the two coefficients are $\geq 2$, we see that $\left|\Delta_{1}\right| \geq 2$ and $\left|\Delta_{2}\right| \geq 2$. In particular, $L \neq G_{2}$. If $L \cong F_{4}$, then we must have $\Delta_{1}$ and $\Delta_{2}$ each isomorphic to the root set of $B_{2}=C_{2}$, and this is not the case by [D, Table 10]. Thus $L \cong E_{6}, E_{7}$ or $E_{8}$, and then all roots have the same length. With this, we see that $\left|\Delta_{1}\right| \geq 4$ and $\left|\Delta_{2}\right| \geq 4$, so $L \cong E_{8}, \Sigma_{\lambda} \cup\left\{\alpha^{\prime}, \beta^{\prime}\right\}=\bar{\Delta}_{1} \cup \bar{\Delta}_{2}$, and $\Delta_{1}$ and $\Delta_{2}$ are simple roots for algebras of type $D_{4}$. Furthermore, it is clear that $\alpha^{\prime}$ must be the central node of $\bar{\Delta}_{1}$, and $\beta^{\prime}$ is the central node of $\bar{\Delta}_{2}$. In particular, by deleting $\alpha^{\prime}$ and $\beta^{\prime}$ from $\bar{\Delta}_{1} \cup \bar{\Delta}_{2}$, we see that $\Sigma_{\lambda}$ is the set of simple roots for an algebra isomorphic to $8 A_{1}$. In other words, $P_{\lambda} \cong 8 A_{1}$ and $\left|\Phi_{\lambda}\right|=16$.

Suppose $\lambda$ has order 2 , and note that $E_{8}$ contains $A_{8}$. Since $A_{8}$ has full rank, its roots $\left\{ \pm\left(e_{i}-e_{j}\right) \mid 1 \leq i<j \leq 9\right\}$ span $V$, and we can extend $\lambda$ to a functional on the vector space $W$ with basis $\left\{e_{1}, e_{2}, \ldots, e_{9}\right\}$ by setting $\lambda\left(e_{k}\right)=0$ for some $k$. Then, for each $i$, we have $\lambda\left(e_{i}\right) \in \mathbb{Z} / 2=\mathbb{Z} \cup(\mathbb{Z}+1 / 2)$, and there are at least five basis elements, say $e_{1}, e_{2}, \ldots, e_{5}$, whose $\lambda$ values are congruent modulo $\mathbb{Z}$. We then obtain at least 20 roots, namely $\pm\left(e_{i}-e_{j}\right)$ with $1 \leq i<j \leq 5$, that are contained in $\Phi_{\lambda}$, a contradiction since $\left|\Phi_{\lambda}\right|=16$. On the other hand, if $\lambda$ has order $>2$, then there is a root $\gamma$ with $\lambda(\gamma)$ having order $>2$, and Lemma 2.2(iii) implies that $P_{\lambda} \neq 8 A_{1}$. Thus $\alpha^{\prime}-\beta^{\prime}$ is indeed a root.

In the next lemma, $\Sigma$ is a set of simple roots of $L$, and $\bar{\Sigma}$ is the extension of $\Sigma$ obtained by adjoining the lowest root. The following result describes a rather standard procedure for counting semisimple subalgebras of $L$. The proof is slightly longer than necessary, to make it somewhat more informative.

Lemma 2.5. Let $P^{\prime}$ be a one-step subalgebra of $L$, with simple roots $\Sigma^{\prime}$ and root system $\Phi^{\prime}$. Assume that

$i$. there are $m_{1}$ distinct sets of simple roots $\Sigma$ of $L$ such that $\Sigma^{\prime}$ is obtained from the completed diagram $\bar{\Sigma}$ by deleting a node.

ii. there are $m_{2}$ distinct sets $\Delta \cong \Sigma^{\prime}$ of roots of $L$ obtained by deleting a node from a fixed $\bar{\Sigma}$. 
If the index of $P^{\prime}$ is defined by $\operatorname{Ind}\left(P^{\prime}\right)=m_{1} / m_{2}$, then the number of one-step subalgebras $P$ of $L$, isomorphic to $P^{\prime}$, is given by

$$
\# P=\frac{|\mathcal{W}(\Phi)|}{\left|\mathcal{W}\left(\Phi^{\prime}\right)\right|} \cdot \frac{1}{\operatorname{Ind}\left(P^{\prime}\right)},
$$

where $\mathcal{W}$ indicates the Weyl group of the root set.

Proof. Let $\mathcal{A}$ denote the collection of all subsets $A$ of the set of roots $\Phi$ of $L$ such that $A \cong \Sigma^{\prime}$ and such that $A$ can be obtained from some $\bar{\Sigma}$ by deleting a node. Furthermore, let $\mathcal{B}$ denote the collection of all simple root subsets for $L$, so that $\mathcal{B}$ is the collection of all such $\Sigma$. The one-step procedure clearly determines multivalued maps from $\mathcal{A}$ to $\mathcal{B}$ and from $\mathcal{B}$ to $\mathcal{A}$. In both cases, these maps are achieved by first adding a root and then deleting a different root. We briefly describe these maps in somewhat more detail.

Let $A \in \mathcal{A}$. Then the roots in $A$ uniquely determine its Dynkin diagram, and from this diagram, we easily see the possible places where a node can be adjoined to form $\bar{\Sigma}$, the completed Dynkin diagram of $L$ for some set $\Sigma$ of simple roots. By assumption, at least one such node exists, but due to certain symmetries, there may be more than one possibility. Since all simple root sets of $L$ are conjugate under the Weyl group $\mathcal{W}(\Phi)$, we can assume that $\Sigma$ is described in the form given by [Bo, Plates I-IX]. In most cases, the set of roots in $A$ is now uniquely determined by the geometry, and if this occurs, then it is a simple matter to check whether the potential roots we wish to adjoin exist or not. Note that any such root is uniquely determined by the diagram, since we know its inner products with the roots in $A$, and these roots form a basis for $V$.

On the other hand, if $A$ is not uniquely determined by the geometry, then we are dealing with $C_{k}+C_{n-k}$ in $C_{n}, D_{k}+D_{n-k}$ in $D_{n}, A_{1}+A_{5}$ in $E_{6}, A_{1}+D_{6}$ in $E_{7}$, or $A_{2}+A_{5}$ in $E_{7}$. Fortunately, in all of these cases, and no matter how $A$ is embedded in $\bar{\Sigma}$, there is a root in the first summand of $A$ which, when deleted from $\bar{\Sigma}$, yields a simple root set isomorphic to $\Sigma$. Thus, we can assume that this deleted set is $\Sigma$, as described above, and that the first summand of $A$ contains the node of $\bar{\Sigma}$ corresponding to the lowest root. With this assumption, the set of roots in $A$ is now uniquely determined and we can proceed as above to test whether the roots we wish to adjoin exist or not.

Of course, once we have obtained a completed Dynkin diagram of $L$, there may be more than one node that can be deleted to yield a member of $\mathcal{B}$. Again, this is due to possible symmetries in the diagram $\bar{\Sigma}$. In any case, we conclude that there exists a fixed parameter $m_{1}$ which counts the number of members of $\mathcal{B}$ that arise from a fixed member of $\mathcal{A}$.

Conversely, if we start with $B \in \mathcal{B}$, then the completed diagram $\bar{B}$ is uniquely determined by adding the lowest root, and there is at least one node that can be deleted to obtain a root set $A \in \mathcal{A}$. Due to symmetries of $\bar{B}$, there may be more than one possibility for $A$ and we let $m_{2}$ be the fixed parameter that counts the number of such possibilities. By computing the size of the set

$$
\{(A, B) \mid A \in \mathcal{A}, B \in \mathcal{B}, A \mapsto B, B \mapsto A\} \subseteq \mathcal{A} \times \mathcal{B}
$$

in two different ways, we see that $|\mathcal{A}| m_{1}=|\mathcal{B}| m_{2}$. Hence $|\mathcal{A}|=|\mathcal{B}| / \operatorname{Ind}\left(P^{\prime}\right)$.

Finally, by $[\mathrm{H}$, Theorem $10.3(\mathrm{~b})(\mathrm{e})],|\mathcal{B}|=|\mathcal{W}(\Phi)|$ and $|\mathcal{A}|=\left|\mathcal{W}\left(\Phi^{\prime}\right)\right| \cdot(\# P)$, where $\# P$ is the number of one-step subalgebras $P$ of $L$ that are isomorphic to $P^{\prime}$. 
With this, we obtain

$$
\# P=\frac{|\mathcal{A}|}{\left|\mathcal{W}\left(\Phi^{\prime}\right)\right|}=\frac{|\mathcal{W}(\Phi)|}{\left|\mathcal{W}\left(\Phi^{\prime}\right)\right|} \cdot \frac{1}{\operatorname{Ind}\left(P^{\prime}\right)},
$$

as required.

\section{Classical Lie algebras}

As we mentioned earlier, the paper $[\mathrm{BP}]$ contains a rather precise description of the maximal functionals in case $L$ is of classical type. Thus, the following proposition is merely a translation of the results of [BP, Section 5] into this new context. Here, the Count column indicates the number of composite functions $\kappa \circ \lambda: \Phi \rightarrow \mathbb{Q} / \mathbb{Z}$ with $P_{\lambda}$ having the appropriate isomorphism type, and it is computed below. This is, of course, the same as the number of $\Lambda_{\Phi^{-}}$-cosets of $\mathfrak{M}$ corresponding to $P_{\lambda}$. On the other hand, the Index column, with each entry written as $m_{1} / m_{2}$, and the $\# P_{\lambda}$ column will be discussed in more detail in Example 3.2. Note that, for classical Lie algebras, the entries in the Count and $\# P_{\lambda}$ columns are identical. As we will see, this is not true in general for the exceptional Lie algebras.

Proposition 3.1. Let $L$ be one of the classical Lie algebras. If $\lambda$ is a maximal functional, then we have

\begin{tabular}{||c|c|c|c|c|c|c||}
\hline \hline Type & Order & $P_{\lambda}$ & Range & Index & $\# P_{\lambda}$ & Count \\
\hline \hline$A_{n}$ & 1 & $A_{n}$ & & & 1 & 1 \\
\hline$B_{n}$ & 1 & $B_{n}$ & & & 1 & 1 \\
& 2 & $B_{k}+D_{n-k}$ & $0 \leq k \leq n-2$ & $2 / 1$ & $\left(\begin{array}{l}n \\
k\end{array}\right)$ & $\left(\begin{array}{l}n \\
k\end{array}\right)$ \\
\hline$C_{n}$ & 1 & $C_{n}$ & & & 1 & 1 \\
& 2 & $C_{k}+C_{n-k}$ & $1 \leq k<n / 2$ & $2 / 2$ & $\left(\begin{array}{l}n \\
k\end{array}\right)$ & $\left(\begin{array}{c}n \\
k\end{array}\right)$ \\
& 2 & $2 C_{k}$ & $k=n / 2$ & $2 / 1$ & $\frac{1}{2}\left(\begin{array}{l}n \\
k\end{array}\right)$ & $\frac{1}{2}\left(\begin{array}{l}n \\
k\end{array}\right)$ \\
\hline$D_{n}$ & 1 & $D_{n}$ & & 1 & 1 \\
& 2 & $D_{k}+D_{n-k}$ & $2 \leq k<n / 2$ & $4 / 2$ & $\left(\begin{array}{l}n \\
k\end{array}\right)$ & $\left(\begin{array}{c}n \\
k\end{array}\right)$ \\
& 2 & $2 D_{k}$ & $k=n / 2$ & $4 / 1$ & $\frac{1}{2}\left(\begin{array}{l}n \\
k\end{array}\right)$ & $\frac{1}{2}\left(\begin{array}{l}n \\
k\end{array}\right)$ \\
\hline \hline
\end{tabular}

where we use $B_{0}=0, B_{1} \cong C_{1} \cong A_{1}, D_{2} \cong 2 A_{1}$ and $D_{3} \cong A_{3}$. In particular, if $P$ is a subalgebra of $L$, then $P=P_{\lambda}$ for some maximal functional $\lambda$ of order 2 if and only if $P$ is a maximal semisimple one-step subalgebra of $L$ of full rank.

Proof. The result is clear if $L$ is of type $A_{n}$. For the remaining types, let $V$ have the orthonormal basis $\Omega=\left\{e_{1}, e_{2}, \ldots, e_{n}\right\}$, and use the description of the root set $\Phi$ as given in [Bo, Plates II-IV]. The arguments in the three cases are similar, but there are essential differences. We are, of course, concerned with rational numbers of order 1 or 2 . These are elements contained in $\mathbb{Z} / 2=\mathbb{Z} \cup(\mathbb{Z}+1 / 2)$. For convenience, we say that the elements $q$ of $\mathbb{Z}$ are even and those of $(\mathbb{Z}+1 / 2)$ are odd. In other words, if $q=a / 2$ with $a \in \mathbb{Z}$, then the parity of $q$ is the same as the usual parity of the integer $a$.

We start with $L \cong B_{n}$, and here we know that the long roots in $\Phi$ consist of all $\pm e_{i} \pm e_{j}$ with $1 \leq i<j \leq n$, while the short roots are the vectors $\pm e_{i}$ for $1 \leq i \leq n$. 
Furthermore, by [BP, Proposition 5.4], $\lambda$ is maximal if and only if $\lambda(\Omega) \subseteq \mathbb{Z} / 2$ and there is no subscript $i_{0}$ such that $\lambda\left(e_{i_{0}}\right) \in \mathbb{Z}+1 / 2$ while $\lambda\left(e_{i}\right) \in \mathbb{Z}$ for the remaining $i \neq i_{0}$. To reinterpret such functionals into the present context, we note that $\kappa \circ \lambda$ is determined by the parity of the various $\lambda\left(e_{i}\right)$. Say $k$ of these are even, so that $n-k$ are odd. There are, of course, $\left(\begin{array}{l}n \\ k\end{array}\right)$ choices for which of the $e_{i}$ s have $\lambda\left(e_{i}\right)$ even, and suppose, for convenience, that $\lambda\left(e_{1}\right), \lambda\left(e_{2}\right), \ldots, \lambda\left(e_{k}\right)$ are even and then that $\lambda\left(e_{k+1}\right), \lambda\left(e_{k+2}\right), \ldots, \lambda\left(e_{n}\right)$ are odd. Now $0 \leq k \leq n$, and $k \neq n-1$ because of the condition on the subscript $i_{0}$ mentioned above. Also note that if $k=n$, then $\lambda$ has order 1 . Thus we can assume that $0 \leq k \leq n-2$. Since $\lambda\left( \pm e_{i} \pm e_{j}\right) \in \mathbb{Z}$ occurs if and only if $1 \leq i<j \leq k$ or $k+1 \leq i<j \leq n$, and since $\lambda\left( \pm e_{i}\right) \in \mathbb{Z}$ if and only if $1 \leq i \leq k$, it follows that

$$
\Phi_{\lambda}=\left\{ \pm e_{i} \pm e_{j}, \pm e_{i} \mid 1 \leq i<j \leq k\right\} \cup\left\{ \pm e_{i} \pm e_{j} \mid k+1 \leq i<j \leq n\right\} .
$$

Hence $P_{\lambda} \cong B_{k}+D_{n-k}$, where $B_{0}=0, B_{1} \cong A_{1}, D_{2} \cong 2 A_{1}$ and $D_{3} \cong A_{3}$.

If $L$ is of type $C_{n}$, then the short roots in $\Phi$ consist of all $\pm e_{i} \pm e_{j}$ with $i<j$ and the long roots are all of the form $\pm 2 e_{i}$. Furthermore, by [BP, Proposition 5.5], $\lambda$ is maximal if and only if $\lambda(\Omega) \subseteq \mathbb{Z} / 2$. Suppose $k$ of the $\lambda\left(e_{i}\right)$ have one parity and the remaining $n-k$ have the other. Then we can assume that $0 \leq k \leq n / 2$. Consider, for example, the situation where $\lambda\left(e_{1}\right), \lambda\left(e_{2}\right), \ldots, \lambda\left(e_{k}\right)$ have one parity, while $\lambda\left(e_{k+1}\right), \lambda\left(e_{k+2}\right), \ldots, \lambda\left(e_{n}\right)$ have the other. If $k=0$, then $\lambda(\Phi) \subseteq \mathbb{Z}$ and $\lambda$ has order 1 . Thus, when $\lambda$ has order 2 , we have $1 \leq k \leq n / 2$ and

$$
\Phi_{\lambda}=\left\{ \pm e_{i} \pm e_{j}, \pm 2 e_{i} \mid 1 \leq i<j \leq k\right\} \cup\left\{ \pm e_{i} \pm e_{j}, \pm 2 e_{i} \mid k+1 \leq i<j \leq n\right\} .
$$

In other words, $P_{\lambda} \cong C_{k}+C_{n-k}$, where $C_{1} \cong A_{1}$. Note that $\kappa \circ \lambda$ is uniquely determined by $\Phi_{\lambda}$, since $\kappa \circ \lambda(\alpha)=1 / 2$ for all $\alpha \in \Phi \backslash \Phi_{\lambda}$. Furthermore, if $k<n / 2$, then there are precisely $\left(\begin{array}{l}n \\ k\end{array}\right)$ choices for those $e_{i}$ s that correspond to this smaller parameter. Hence the Count here is $\left(\begin{array}{l}n \\ k\end{array}\right)$. On the other hand, when $n$ is even and $k=n / 2$, then $\left(\begin{array}{l}n \\ k\end{array}\right)$ clearly double counts the number of these choices.

Finally, if $L$ is of type $D_{n}$, then the roots in $\Phi$ all have the same length and are given by $\pm e_{i} \pm e_{j}$ with $i<j$. Furthermore, by [BP, Proposition 5.6], $\lambda$ is maximal if and only if $\lambda(\Omega) \subseteq \mathbb{Z} / 2$ and there are no subscripts $i_{0}$ with $\lambda\left(e_{i_{0}}\right)$ having parity different from that of the remaining $\lambda\left(e_{i}\right)$. It follows that, for example, if $\lambda\left(e_{1}\right), \lambda\left(e_{2}\right), \ldots, \lambda\left(e_{k}\right)$ have one parity, while $\lambda\left(e_{k+1}\right), \lambda\left(e_{k+2}\right), \ldots, \lambda\left(e_{n}\right)$ have the other, then $k=0$ or $2 \leq k \leq n / 2$. Now if $k=0$, then $\lambda(\Phi) \subseteq \mathbb{Z}$, and $\lambda$ has order 1 . Thus, we can assume that $2 \leq k \leq n-2$ and that $\lambda$ has order 2 . Here it is again easy to determine $\Phi_{\lambda}$, and we find that $P_{\lambda} \cong D_{k}+D_{n-k}$, where $D_{2} \cong 2 A_{1}$ and $D_{3} \cong A_{3}$. The result now follows as above.

It remains to consider the maximality of the various $P_{\lambda}$ with $\lambda$ of order 2 . To this end, let $P$ be a semisimple subalgebra of $L$ properly containing $P_{\lambda}$ and let $\Gamma \subseteq \Phi$ be its root system. If $\alpha \in \Gamma \backslash \Phi_{\lambda}$ and $\beta \in \Phi \backslash \Phi_{\lambda}$, then, since $P_{\lambda}$ is isomorphic to a one-step subalgebra of $L$, Lemma 2.4 implies that either $\alpha^{\prime}=\beta^{\prime}$ or $\alpha^{\prime}-\beta^{\prime} \in \Phi$. In the latter case, since $\lambda(\alpha) \equiv 1 / 2 \equiv \lambda(\beta) \bmod \mathbb{Z}$, Lemma 2.1(iii) implies that $\lambda\left(\alpha^{\prime}-\beta^{\prime}\right)=\lambda\left(\alpha^{\prime}\right)-\lambda\left(\beta^{\prime}\right) \equiv 0 \bmod \mathbb{Z}$ and hence that $\alpha^{\prime}-\beta^{\prime} \in \Phi_{\lambda} \subseteq \Gamma$. It now follows from Lemma 2.1(i)(ii) that $\alpha^{\prime}, \beta^{\prime}$ and then $\beta$ are all contained in $\Gamma$. In other words, $\Gamma=\Phi$ and hence $P=L$. Thus $P_{\lambda}$ is indeed maximal, and we conclude from [D, Theorem 5.3] that $P_{\lambda}$ is a one-step subalgebra of $L$.

Next, we discuss how the computational techniques of Lemma 2.5 apply to the classical Lie algebras considered above. 
Example 3.2. For any Lie algebra $L$, there is of course one $\kappa \circ \lambda$ corresponding to a maximal functional of order 1 . Thus, in studying the classical Lie algebras, we can assume that $L$ is of type $B_{n}, C_{n}$ or $D_{n}$, and that $\lambda$ has order 2 . In this case, we know from Proposition 3.1 that each $P_{\lambda}$ is a maximal one-step semisimple subalgebra of $L$ of full rank. Furthermore, since these functionals have order 2 , it is clear that there is a one-to-one correspondence between the various $P_{\lambda}$ and the composite functions $\kappa \circ \lambda$. In the following computations, we will first ignore the triple symmetry of the Dynkin diagram for $D_{4}$. Later on, we will show that this symmetry causes no difficulties.

We use [Bo, Plates II-IV] throughout these arguments and we start with $L \cong B_{n}$. Here the one-step subalgebras are all isomorphic to $B_{k}+D_{n-k}$ with $0 \leq k \leq n-2$, and it is clear that $m_{1}=2$ and $m_{2}=1$. Thus $\operatorname{Ind}\left(P_{\lambda}\right)=2 / 1$ and, by Lemma 3.2, the number of $\kappa \circ \lambda$, with $P_{\lambda}$ isomorphic to the above subalgebra with parameter $k$, is given by

$$
\# P_{\lambda}=\frac{|\mathcal{W}(\Phi)|}{\left|\mathcal{W}\left(\Phi_{\lambda}\right)\right|} \cdot \frac{1}{\operatorname{Ind}\left(P_{\lambda}\right)}=\frac{2^{n} n !}{2^{k} k ! \cdot 2^{n-k-1}(n-k) ! \cdot 2}=\left(\begin{array}{l}
n \\
k
\end{array}\right) .
$$

Next, let $L \cong C_{n}$, so the one-step subalgebras are each isomorphic to $C_{k}+C_{n-k}$ with $1 \leq k \leq n / 2$. Again, it is easy to see that $m_{1}=2$, and that $m_{2}=2$ if $k \neq n / 2$. On the other hand, $m_{2}=1$ when $k=n / 2$. Thus $\operatorname{Ind}\left(P_{\lambda}\right)=2 / 2$ in the first case, and $2 / 1$ in the second, and the number of composite functions $\kappa \circ \lambda$, with $P_{\lambda}$ isomorphic to the subalgebra with parameter $k \neq n / 2$, is given by

$$
\# P_{\lambda}=\frac{|\mathcal{W}(\Phi)|}{\left|\mathcal{W}\left(\Phi_{\lambda}\right)\right|} \cdot \frac{1}{\operatorname{Ind}\left(P_{\lambda}\right)}=\frac{2^{n} n !}{2^{k} k ! \cdot 2^{n-k}(n-k) ! \cdot 1}=\left(\begin{array}{l}
n \\
k
\end{array}\right) .
$$

If $k=n / 2$, we obtain $\frac{1}{2}\left(\begin{array}{l}n \\ k\end{array}\right)$, as expected.

Finally, let $L \cong D_{n}$. Then $P_{\lambda} \cong D_{k}+D_{n-k}$ with $2 \leq k \leq n / 2$, and we have $m_{1}=4$ and $m_{2}=2$ if $k \neq n / 2$. When $k=n / 2$, then $m_{2}=1$. Thus $\operatorname{Ind}\left(P_{\lambda}\right)=4 / 2$ in the former case and $4 / 1$ in the latter. Again, by Lemma 3.2, the number of $\kappa \circ \lambda$, with $P_{\lambda}$ isomorphic to the subalgebra with parameter $k \neq n / 2$, is given by

$$
\# P_{\lambda}=\frac{|\mathcal{W}(\Phi)|}{\left|\mathcal{W}\left(\Phi_{\lambda}\right)\right|} \cdot \frac{1}{\operatorname{Ind}\left(P_{\lambda}\right)}=\frac{2^{n-1} n !}{2^{k-1} k ! \cdot 2^{n-k-1}(n-k) ! \cdot 2}=\left(\begin{array}{l}
n \\
k
\end{array}\right)
$$

If $k=n / 2$, we again obtain $\frac{1}{2}\left(\begin{array}{l}n \\ k\end{array}\right)$.

Since these numbers agree with the Count given in Proposition 3.1, the symmetry of $D_{4}$ will surely cause no difficulty. To verify this fact in somewhat more generality, let us first consider the subalgebra $B_{n-k}+D_{k}$ in $B_{n}$. We can assume that the simple roots in $D_{k}$ are $-\delta, \alpha_{1}, \alpha_{2}, \ldots, \alpha_{k-1}$, while those in $B_{n-k}$ are $\alpha_{k+1}, \alpha_{k+2}, \ldots, \alpha_{n}$. Here $\alpha_{1}=e_{1}-e_{2}, \alpha_{2}=e_{2}-e_{3}, \ldots, \alpha_{n-1}=e_{n-1}-e_{n}$, and $\alpha_{n}=e_{n}$. Furthermore, $\delta$ is the highest root, so $-\delta=-e_{1}-e_{2}$. Now $D_{k}$ involves the basis elements $e_{1}, e_{2}, \ldots, e_{k}$, while $B_{n-k}$ involves $e_{k+1}, e_{k+2}, \ldots, e_{n}$. Thus the connecting node $\beta$ must be of the form $\pm e_{i} \pm e_{j}$ with $i \leq k$ and $k+1 \leq j$. Since $\alpha_{1}=e_{1}-e_{2}$ and $-\delta=-e_{1}-e_{2}$, we see that if $\beta$ is orthogonal to either of these, it will be orthogonal to the other. Thus $\beta$ must connect to $\alpha_{k-1}$. Similarly, $\alpha_{n}=e_{n}$ and $\alpha_{n-1}=e_{n-1}-e_{n}$, so if $\beta$ is orthogonal to $\alpha_{n-1}$, then it is orthogonal to $\alpha_{n}$. Thus $\beta$ must connect to $\alpha_{k+1}$ and, since $\beta$ is uniquely determined by its position in the extended Dynkin diagram, only $\beta=\alpha_{k}$ can give rise to the appropriate diagram.

The argument with $D_{k}+D_{n-k}$ in $D_{n}$ is essentially the same. Again, we can assume that the simple roots in $D_{k}$ are $-\delta, \alpha_{1}, \alpha_{2}, \ldots, \alpha_{k-1}$, while those in $D_{n-k}$ are 
given by $\alpha_{k+1}, \alpha_{k+2}, \ldots, \alpha_{n}$. Here $\alpha_{1}=e_{1}-e_{2}, \alpha_{2}=e_{2}-e_{3}, \ldots, \alpha_{n-1}=e_{n-1}-e_{n}$, and $\alpha_{n}=e_{n-1}+e_{n}$. Furthermore, $\delta$ is the highest root, so $-\delta=-e_{1}-e_{2}$. We now apply the same proof as above, but with one change. Namely, we note that if $\beta$ is orthogonal to either $\alpha_{n-1}$ or $\alpha_{n}$, then it is orthogonal to both. With this, we conclude that $\beta$ must be connected to $\alpha_{k-1}$ and $\alpha_{k+1}$, and hence only $\beta=\alpha_{k}$ can give rise to the completed Dynkin diagram of $D_{n}$.

\section{Exceptional Lie algebras}

We would like to obtain the same sort of results for the exceptional Lie algebras as were obtained for the classical ones in the preceding section. To this end, we start with

Lemma 4.1. Let $L$ be an exceptional Lie algebra and let $\lambda: V \rightarrow \mathbb{R}$ be a maximal functional. If $\Gamma$ is a root system in $\Phi$ with $\Gamma \supseteq \Phi_{\lambda}$, then $\kappa \circ \lambda(\Gamma)$ is a finite subgroup of $\mathbb{Q} / \mathbb{Z}$ and $\Gamma=\{\alpha \in \Phi \mid \kappa \circ \lambda(\alpha) \in \kappa \circ \lambda(\Gamma)\}$. Furthermore, $\kappa \circ \lambda(\Gamma)$ is cyclic, and the possible orders for $\lambda$ are given by

\begin{tabular}{||l|l||}
\hline \hline Type & Order \\
\hline \hline$E_{6}$ & $1,2,3$ \\
$E_{7}$ & $1,2,3,4$ \\
$E_{8}$ & $1,2,3,4,5,6$ \\
$F_{4}$ & $1,2,3,4$ \\
$G_{2}$ & $1,2,3$ \\
\hline \hline
\end{tabular}

Proof. We prove that $\kappa \circ \lambda(\Gamma)$ is a group. Since $\kappa \circ \lambda(\Gamma) \supseteq \kappa \circ \lambda\left(\Phi_{\lambda}\right)=0$, it suffices to show that if $\alpha, \beta \in \Gamma$ and if $\kappa \circ \lambda(\alpha) \neq \kappa \circ \lambda(\beta)$, then $\kappa \circ \lambda(\alpha)-\kappa \circ \lambda(\beta) \in \kappa \circ \lambda(\Gamma)$. To this end, note that $\kappa \circ \lambda\left(\alpha^{\prime}\right)=\kappa \circ \lambda(\alpha), \kappa \circ \lambda\left(\beta^{\prime}\right)=\kappa \circ \lambda(\beta)$, and $\alpha^{\prime}, \beta^{\prime} \in \Gamma$. Since $\alpha^{\prime} \neq \beta^{\prime}$, we conclude from Lemma 2.4 that $\alpha^{\prime}-\beta^{\prime} \in \Phi$ and hence $\alpha^{\prime}-\beta^{\prime} \in \Gamma$, because $\Gamma$ is a root system in $\Phi$. But then $\kappa \circ \lambda(\Gamma)$ contains

$$
\kappa \circ \lambda\left(\alpha^{\prime}-\beta^{\prime}\right)=\kappa \circ \lambda\left(\alpha^{\prime}\right)-\kappa \circ \lambda\left(\beta^{\prime}\right)=\kappa \circ \lambda(\alpha)-\kappa \circ \lambda(\beta),
$$

as required. Thus $\kappa \circ \lambda(\Gamma)$ is a finite subgroup of the locally cyclic group $\mathbb{Q} / \mathbb{Z}$, and therefore it is cyclic.

Now suppose $\alpha \in \Phi$ satisfies $\kappa \circ \lambda(\alpha) \in \kappa \circ \lambda(\Gamma)$. Then there exists $\beta \in \Gamma$ with $\kappa \circ \lambda(\alpha)=\kappa \circ \lambda(\beta)$ and, by Lemma 2.4, either $\alpha^{\prime}=\beta^{\prime}$ or $\alpha^{\prime}-\beta^{\prime} \in \Phi$. In the latter situation,

$$
\kappa \circ \lambda\left(\alpha^{\prime}-\beta^{\prime}\right)=\kappa \circ \lambda\left(\alpha^{\prime}\right)-\kappa \circ \lambda\left(\beta^{\prime}\right)=\kappa \circ \lambda(\alpha)-\kappa \circ \lambda(\beta)=0,
$$

so $\alpha^{\prime}-\beta^{\prime} \in \Phi_{\lambda} \subseteq \Gamma$. But $\beta^{\prime} \in \Gamma$, so we conclude in either case that $\alpha^{\prime} \in \Gamma$ and hence that $\alpha \in \bar{\Gamma}$. Finally, by taking $\Gamma=\Phi$, we see that $\kappa \circ \lambda(\Phi)$ is cyclic. In particular, there exists $\gamma \in \Phi$ with the order of $\lambda$ equal to the order of $\lambda(\gamma)$, and therefore Lemma 2.3 yields the result.

It follows from the above table that $e=f$, in the notation of [BP, Lemma 6.1]. We now come to the main result of this paper. Again, Count indicates the number of composite maps $\kappa \circ \lambda$, where $P_{\lambda}$ has the appropriate isomorphism type. This is, of course, the same as the number of $\Lambda_{\Phi}$-cosets of $\mathfrak{M}$ corresponding to $P_{\lambda}$. Furthermore, the Index is described as $m_{1} / m_{2}$. 
Theorem 4.2. Let $L$ be a finite-dimensional exceptional simple Lie algebra over the complex numbers $\mathbb{C}$ and let $\lambda: V \rightarrow \mathbb{R}$ be a maximal (or, rigid) functional of order $c$. Then $P_{\lambda}$ is a one-step subalgebra of $L$ containing the Cartan subalgebra $L_{0}$, and its isomorphism type is obtained from the completed Dynkin diagram of $L$ by deleting a node with corresponding coefficient equal to $c$. We therefore have the following possibilities.

\begin{tabular}{||c|c|c|c|c|c||}
\hline \hline Type & Order & $P_{\lambda}$ & Index & $\# P_{\lambda}$ & Count \\
\hline \hline \multirow{2}{*}{$E_{6}$} & 1 & $E_{6}$ & & 1 & 1 \\
& 2 & $A_{5}+A_{1}$ & $3 / 3$ & $2^{2} \cdot 3^{2}$ & $2^{2} \cdot 3^{2}$ \\
& 3 & $3 A_{2}$ & $6 / 1$ & $2^{3} \cdot 5$ & $2^{4} \cdot 5$ \\
\hline$E_{7}$ & 1 & $E_{7}$ & & 1 & 1 \\
& 2 & $A_{7}$ & $2 / 1$ & $2^{2} \cdot 3^{2}$ & $2^{2} \cdot 3^{2}$ \\
& 2 & $D_{6}+A_{1}$ & $2 / 2$ & $3^{2} \cdot 7$ & $3^{2} \cdot 7$ \\
& 3 & $A_{5}+A_{2}$ & $4 / 2$ & $2^{4} \cdot 3 \cdot 7$ & $2^{5} \cdot 3 \cdot 7$ \\
& 4 & $2 A_{3}+A_{1}$ & $4 / 1$ & $2 \cdot 3^{2} \cdot 5 \cdot 7$ & $2^{2} \cdot 3^{2} \cdot 5 \cdot 7$ \\
\hline \multirow{6}{*}{$E_{8}$} & 1 & $E_{8}$ & & 1 & 1 \\
& 2 & $D_{8}$ & $1 / 1$ & $3^{3} \cdot 5$ & $3^{3} \cdot 5$ \\
& 2 & $E_{7}+A_{1}$ & $1 / 1$ & $2^{3} \cdot 3 \cdot 5$ & $2^{3} \cdot 3 \cdot 5$ \\
& 3 & $A_{8}$ & $2 / 1$ & $2^{6} \cdot 3 \cdot 5$ & $2^{7} \cdot 3 \cdot 5$ \\
& 3 & $E_{6}+A_{2}$ & $2 / 1$ & $2^{5} \cdot 5 \cdot 7$ & $2^{6} \cdot 5 \cdot 7$ \\
& 4 & $A_{7}+A_{1}$ & $2 / 1$ & $2^{5} \cdot 3^{3} \cdot 5$ & $2^{6} \cdot 3^{3} \cdot 5$ \\
& 4 & $D_{5}+A_{3}$ & $2 / 1$ & $2^{3} \cdot 3^{3} \cdot 5 \cdot 7$ & $2^{4} \cdot 3^{3} \cdot 5 \cdot 7$ \\
& 5 & $2 A_{4}$ & $4 / 1$ & $2^{6} \cdot 3^{3} \cdot 7$ & $2^{8} \cdot 3^{3} \cdot 7$ \\
& 6 & $A_{5}+A_{2}+A_{1}$ & $2 / 1$ & $2^{7} \cdot 3^{2} \cdot 5 \cdot 7$ & $2^{8} \cdot 3^{2} \cdot 5 \cdot 7$ \\
\hline \multirow{6}{*}{$F_{4}$} & 1 & $F_{4}$ & & 1 & 1 \\
& 2 & $B_{4}$ & $1 / 1$ & 3 & 3 \\
& 2 & $C_{3}+A_{1}$ & $1 / 1$ & $2^{2} \cdot 3$ & $2^{2} \cdot 3$ \\
& 3 & $2 A_{2}$ & $2 / 1$ & $2^{4}$ & $2^{5}$ \\
& 4 & $B_{3}+A_{1}$ & $1 / 1$ & $2^{2} \cdot 3$ & $2^{3} \cdot 3$ \\
\hline \multirow{6}{*}{$G_{2}$} & 1 & $G_{2}$ & & 1 & 1 \\
& 2 & $2 A_{1}$ & $1 / 1$ & 3 & 3 \\
& 3 & $A_{2}$ & $2 / 1$ & 1 & 2 \\
\hline \hline
\end{tabular}

Here, the Count is given by

$$
\# \kappa \circ \lambda=\left(\# P_{\lambda}\right) \cdot \phi(c)=\frac{|\mathcal{W}(\Phi)|}{\left|\mathcal{W}\left(\Phi_{\lambda}\right)\right|} \cdot \frac{\phi(c)}{\operatorname{Ind}\left(P_{\lambda}\right)} .
$$

Furthermore, there is a natural one-to-one correspondence between the semisimple subalgebras $M$ of full rank with $L \supseteq M \supseteq P_{\lambda}$ and the subgroups of $\kappa \circ \lambda(\Phi) \cong \mathbb{Z} / c \mathbb{Z}$. In particular, $P_{\lambda}$ is a maximal semisimple subalgebra of full rank if and only if $\lambda$ has prime order. Finally, any one-step subalgebra of $L$ is a suitable $P_{\lambda}$.

Proof. We can assume that $c \neq 1$. If $T$ is a subgroup of $\mathbb{Z} / c \mathbb{Z}$, then it is easy to see that $\Theta=\{\alpha \in \Phi \mid \kappa \circ \lambda(\alpha) \in T\}$ is a root set in $\Phi$, containing $\Phi_{\lambda}$. Furthermore, by Lemma 4.1, it follows first that $\kappa \circ \lambda(\Theta)=T$ and then that we have a natural one-to-one correspondence between the semisimple subalgebras $P$ of $L$ with $P \supseteq P_{\lambda}$ 
and the subgroups of $\mathbb{Z} / c \mathbb{Z}$. Thus the number of such subalgebras is precisely equal to the number of divisors of $c$. Now choose a root $\alpha \in \Phi$ so that $\kappa \circ \lambda(\alpha)$ generates the cyclic group $\kappa \circ \lambda(\Phi)$. In particular, if $\Gamma$ is the root set generated by $\Sigma_{\lambda}$ and $\alpha$, then $\kappa \circ \lambda(\Gamma)=\kappa \circ \lambda(\Phi)$, and hence the one-to-one correspondence implies that $\Gamma=\Phi$. It now follows from Lemma 2.2 that there exists a set $\Sigma=\left\{\alpha_{1}, \alpha_{2}, \ldots, \alpha_{n}\right\}$ of simple roots of $\Phi$, with highest root $\delta=c_{1} \alpha_{1}+c_{2} \alpha_{2}+\cdots+c_{n} \alpha_{n}$, such that, for some subscript $j$, we have $c \mid c_{j}$. Furthermore, $\Sigma_{\lambda}$ consists of $(-\delta)$ and those simple roots $\alpha_{i}$ with $i \neq j$.

It remains to show that $c=c_{j}$. To this end, define a linear functional $\mu: V \rightarrow \mathbb{R}$ by setting $\mu\left(\alpha_{i}\right)=0$ if $i \neq j$ and $\mu\left(\alpha_{j}\right)=1 / c_{j}$. Then $\mu(\delta)=1 \in \mathbb{Z}$, so $\mu$ is a maximal functional with $\Phi_{\mu} \supseteq \Sigma_{\lambda}$, and hence $\Phi_{\mu} \supseteq \Phi_{\lambda}$. In particular, $P_{\mu} \supseteq P_{\lambda}$ and the order of $\mu$ is precisely equal to $c_{j}$. Now, the number of semisimple subalgebras $P$ of full rank with $L \supseteq P \supseteq P_{\mu}$ is equal to the number of divisors of $c_{j}$, while the number with $L \supseteq P \supseteq P_{\lambda}$ is equal to the number of divisors of $c$. Since $c \mid c_{j}$ and $P_{\mu} \supseteq P_{\lambda}$, we conclude that $P_{\mu}=P_{\lambda}$ and that $c=c_{j}$, as required. It is now a simple matter to determine the possibilities for $\lambda$ and $P_{\lambda}$ from the information in [Bo, Plates V-IX].

The Count computations are easily done by hand using the orders of the Weyl groups, as given in [Bo, Plates I-IX], and the geometry of the standard and completed Dynkin diagrams, which affect the index. Furthermore, $c$ also comes into play in this count, but in a rather simple manner. To start with, we easily verify, by checking coefficients in the highest root, that isomorphic $P_{\lambda}$ s correspond to functionals of the same order. Next, if $\Sigma_{\lambda}$ is obtained from $\bar{\Sigma}$ by deleting a simple root $\alpha$, then $\kappa \circ \lambda\left(\Phi_{\lambda}\right)=0$ implies that $\kappa \circ \lambda$ is uniquely determined by its value on $\alpha$. Indeed, this value must be a generator of the cyclic group $\mathbb{Z} / c \mathbb{Z}$ and, by replacing $\lambda$ by its integer multiple $s \lambda$ with $\operatorname{gcd}(s, c)=1$, we see that $\kappa \circ \lambda(\alpha)$ can be equal to any of these $\phi(c)$ generators. Thus, the number of different composite functions $\kappa \circ \lambda$ with $P_{\lambda}$ having a fixed isomorphism class is precisely equal to $\# P_{\lambda}$, the number of such subalgebras, times $\phi(c)$, where $\phi$ is, of course, the Euler function. Lemma 2.5 now yields the result.

We remark that there is a secondary computer check of these Count values. Specifically, for each $L$ and for each $c$, we use Maple 9, as in [BP, Section 6], to determine the total number of $\kappa \circ \lambda$ functions having order $c$, and these totals do indeed agree with the sum of the values in the table. Maple 9 worksheets, in text readable format, for each of the exceptional Lie algebras can be found on the internet at www.math.wisc.edu/ ${ }^{\sim}$ passman/abstracts.html.

Some sample computations are offered in the following three examples.

Example 4.3. The completed Dynkin diagram of $E_{6}$ is given by

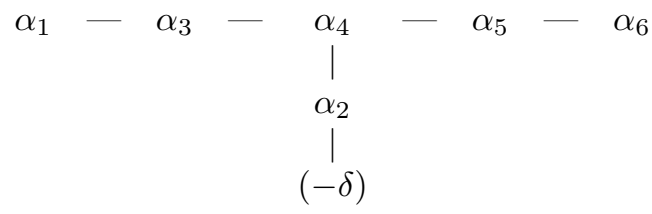

where $\alpha_{1}=\frac{1}{2}\left(f+e_{1}-e_{2}-e_{3}-e_{4}-e_{5}\right), \alpha_{2}=e_{1}+e_{2}, \alpha_{3}=e_{2}-e_{1}, \alpha_{4}=e_{3}-e_{2}$, $\alpha_{5}=e_{4}-e_{3}$ and $\alpha_{6}=e_{5}-e_{4}$. Here $f=e_{8}-e_{7}-e_{6}$ and $\delta=\frac{1}{2}\left(f+e_{1}+e_{2}+e_{3}+e_{4}+e_{5}\right)$. Note that, by deleting any one of $\alpha_{1}, \alpha_{6}$ or $-\delta$, we obtain a simple root system for $L$, and therefore $[\mathrm{H}$, Theorem 10.3(b)(e)] easily implies that the Weyl group $\mathcal{W}(\Phi)$ 
has a subgroup isomorphic to $\mathrm{Sym}_{3}$ that fixes $\alpha_{4}$ and permutes the three vanes. In particular, all three vanes behave in the same manner.

Now suppose that $\lambda$ is a maximal functional of order 3 . Then $P_{\lambda} \cong 3 A_{2}$ and we can assume that $\Sigma_{\lambda}=\left\{\alpha_{3}, \alpha_{1}\right\} \cup\left\{\alpha_{5}, \alpha_{6}\right\} \cup\left\{\alpha_{2},-\delta\right\}$. There are clearly at most $2^{3}=8$ ways of connecting these three vanes to a center node. First, there is the original situation given above, then there are three possibilities obtained by flipping the two roots in one of the vanes; there are three possibilities obtained by flipping the roots in two of the vanes, and finally one possibility if all three vanes are flipped. Suppose the vanes containing $\alpha_{2}$ and $\alpha_{3}$ are both flipped, with the third vane possibly flipped. If $\beta$ is the new central node, then $\beta \perp \alpha_{2}$ and $\beta \perp \alpha_{3}$. Thus $\beta$ is orthogonal to both $e_{1}$ and $e_{2}$, so $\beta= \pm e_{i} \pm e_{j}$ with $i, j \in\{3,4,5\}$. If the third vane is not flipped, then $\beta \perp \alpha_{6}$, so $\beta= \pm\left(e_{4}+e_{5}\right)$ and this contradicts $\left(\beta, \alpha_{1}\right)<0$ and $\left(\beta, \alpha_{5}\right)<0$. On the other hand, if the third vane is flipped, then $\beta \perp \alpha_{5}$, so $\beta= \pm\left(e_{3}+e_{4}\right)$ and it is easy to check that $\beta=e_{3}+e_{4}$ yields the necessary inequalities $\left(\beta, \alpha_{1}\right)<0,(\beta,-\delta)<0$ and $\left(\beta, \alpha_{6}\right)<0$.

We conclude that the triple flip exists but, by symmetry, no double flip can exist. Furthermore, if a single flip exists, then all such single flips exists, and a product of two of these yields a double flip, a contradiction. It follows that there are precisely two ways to adjoin a node to $\Sigma_{\lambda}$ to obtain $\bar{\Sigma}$, the completed diagram of a simple root set $\Sigma$. Of course, once we obtain $\bar{\Sigma}$, there are three possible nodes to delete to obtain $\Sigma$. Thus $m_{1}=2 \cdot 3=6$. On the other hand, it is clear that $m_{2}=1$. Thus $\operatorname{Ind}\left(P_{\lambda}\right)=m_{1} / m_{2}=6$ and the number of composite functions is given by

$$
\# \kappa \circ \lambda=\frac{|\mathcal{W}(\Phi)|}{\left|\mathcal{W}\left(\Phi_{\lambda}\right)\right|} \cdot \frac{\phi(c)}{\operatorname{Ind}\left(P_{\lambda}\right)}=\frac{\left(2^{7} \cdot 3^{4} \cdot 5\right) \cdot 2}{(2 \cdot 3)^{3} \cdot 6}=2^{4} \cdot 5
$$

Example 4.4. The completed Dynkin diagram for $E_{7}$ is given by

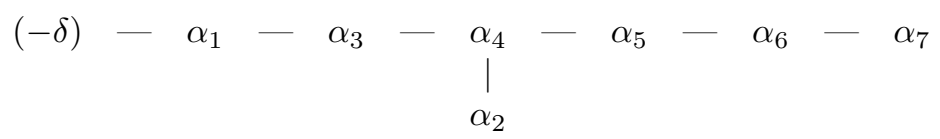

where $\alpha_{1}=\frac{1}{2}\left(e_{1}-e_{2}-e_{3}-e_{4}-e_{5}-e_{6}-f\right), \alpha_{2}=e_{1}+e_{2}, \alpha_{3}=e_{2}-e_{1}, \alpha_{4}=e_{3}-e_{2}$, $\alpha_{5}=e_{4}-e_{3}, \alpha_{6}=e_{5}-e_{4}, \alpha_{7}=e_{6}-e_{5}$, and $f=e_{7}-e_{8}=-\delta$. We consider the linear functionals $\lambda$ of order 3 obtained by deleting the node $\alpha_{3}$ or $\alpha_{5}$ since, in either case, $P_{\lambda} \cong A_{5}+A_{2}$. Note that for each of the two embeddings of $\Sigma_{\lambda}$ in the displayed diagram, there is a root in the $A_{5}$ part that, when deleted from $\bar{\Sigma}$, yields a simple root set isomorphic to $\Sigma$. Thus, we can assume that this deleted set is $\Sigma$, as described above, and that the $A_{5}$ subset of $\Sigma_{\lambda}$ contains the node corresponding to the lowest root. In other words, $\Sigma_{\lambda}=\left\{-\delta, \alpha_{1}, \alpha_{2}, \alpha_{3}, \alpha_{4}\right\} \cup\left\{\alpha_{6}, \alpha_{7}\right\}$, where the first subset corresponds to $A_{5}$ and the second to $A_{2}$.

If $\beta$ is a root in $\Phi$ that connects these two subsets to form a completed Dynkin diagram for $E_{7}$, then $\beta$ connects to $\alpha_{1}$ or $\alpha_{4}$ in the first subset, and to $\alpha_{6}$ or $\alpha_{7}$ in the second. In particular, $\beta$ is orthogonal to $-\delta, \alpha_{2}$ and $\alpha_{3}$, so $\beta$ is orthogonal to $f, e_{1}$ and $e_{2}$, and hence $\beta= \pm e_{i} \pm e_{j}$ with $3 \leq j<i \leq 6$.

Suppose first that $\beta$ connects to $\alpha_{1}$ in the $A_{5}$ part. Then $\beta \perp \alpha_{4}$ and $\left(\beta, \alpha_{1}\right)<0$ easily imply that $\beta=e_{i}+e_{j}$ with $4 \leq j<i \leq 6$. On the $A_{2}$ side, if $\beta$ connects to $\alpha_{6}$, then $\beta$ is orthogonal to $\alpha_{7}=e_{6}-e_{5}$. Thus $\beta=e_{6}+e_{5}$, a contradiction since $\left(e_{6}+e_{5}, \alpha_{6}\right)>0$. On the other hand, if $\beta$ connects to $\alpha_{7}$, then $\beta$ is orthogonal to $\alpha_{6}=e_{5}-e_{4}$ and hence $\beta=e_{5}+e_{4}$. Here, we have $\left(\beta, \alpha_{7}\right)<0$, so $\beta$ is indeed a solution in this case. 
Conversely, if $\beta$ connects to $\alpha_{4}$ in the $A_{5}$ part, then $\left(\beta, \alpha_{4}\right)<0$ implies that $j=3$ and then that $\beta= \pm e_{i}-e_{3}$. Furthermore, since $\beta \perp \alpha_{1}$, we get $\beta=e_{i}-e_{3}$ with $i=4,5$ or 6 . On the $A_{2}$ side, if $\beta$ connects to $\alpha_{6}$, then $\beta$ is orthogonal to $\alpha_{7}=e_{6}-e_{5}$ and hence $\beta=e_{4}-e_{3}=\alpha_{4}$, the original connecting node. On the other hand, if $\beta$ connects to $\alpha_{7}$, then $\beta \perp \alpha_{6}$ and $\left(\beta, \alpha_{7}\right)<0$ yield a contradiction. Thus, again, there is just one solution in this case.

We conclude that there are two embeddings of a fixed $\Sigma_{\lambda}$ into a completed diagram for $E_{7}$, and since each $\bar{\Sigma}$ determines two possible $\Sigma$ s, we see that $m_{1}=$ $2 \cdot 2=4$. Finally, $m_{2}$ is equal to 2 , so we have $\operatorname{Ind}\left(P_{\lambda}\right)=m_{1} / m_{2}=4 / 2$. In particular, since $c=3$, Theorem 4.2 implies that the number of composite functions $\kappa \circ \lambda$ is given by

$$
\# \kappa \circ \lambda=\frac{|\mathcal{W}(\Phi)|}{\left|\mathcal{W}\left(\Phi_{\lambda}\right)\right|} \cdot \frac{\phi(c)}{\operatorname{Ind}\left(P_{\lambda}\right)}=\frac{\left(2^{10} \cdot 3^{4} \cdot 5 \cdot 7\right) \cdot 2}{(6 ! \cdot 3 !) \cdot 2}=2^{5} \cdot 3 \cdot 7
$$

Example 4.5. The completed Dynkin diagram for $E_{8}$ is given by

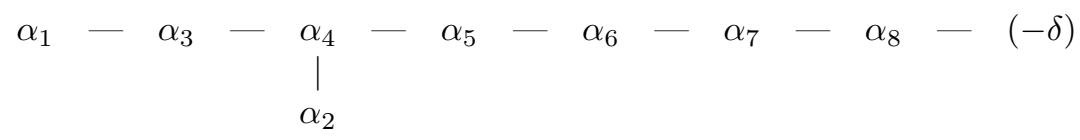

where $\alpha_{1}=\frac{1}{2}\left(e_{1}-e_{2}-e_{3}-e_{4}-e_{5}-e_{6}-e_{7}+e_{8}\right), \alpha_{2}=e_{1}+e_{2}, \alpha_{3}=e_{2}-e_{1}$, $\alpha_{4}=e_{3}-e_{2}, \alpha_{5}=e_{4}-e_{3}, \alpha_{6}=e_{5}-e_{4}, \alpha_{7}=e_{6}-e_{5}, \alpha_{8}=e_{7}-e_{6}$, and $\delta=e_{7}+e_{8}$. We consider the linear functionals $\lambda$ of order 4 obtained by deleting the node $\alpha_{6}$. Thus $P_{\lambda} \cong D_{5}+A_{3}$ and $\Sigma_{\lambda}=\left\{\alpha_{1}, \alpha_{2}, \alpha_{3}, \alpha_{4}, \alpha_{5}\right\} \cup\left\{\alpha_{7}, \alpha_{8},-\delta\right\}$, where the first subset corresponds to $D_{5}$ and the second to $A_{3}$. If $\beta$ is a root in $\Phi$ that connects these two subsets to form a completed Dynkin diagram for $E_{8}$, then $\beta$ connects to $\alpha_{2}$ or $\alpha_{5}$ in the first subset and to $\alpha_{7}$ or $-\delta$ in the second.

Suppose first that $\beta$ connects to $\alpha_{5}$. Then $\beta$ is orthogonal to $\alpha_{2}, \alpha_{3}$ and $\alpha_{4}$, so it is orthogonal to $e_{1}, e_{2}$ and $e_{3}$, and from the nature of the possible roots, we see that $\beta= \pm e_{i} \pm e_{j}$ with $4 \leq j<i$. Furthermore, $\left(\alpha_{5}, \beta\right)<0$, so $j=4$ and $\beta= \pm e_{i}-e_{4}$ for some $i \geq 5$. On the other side, $\beta \perp \alpha_{8}$ implies that $i \neq 6$ or 7 . If $i=5$, then $\beta \perp \alpha_{1}$ implies that $\beta=e_{5}-e_{4}=\alpha_{6}$, the original connecting node. If $i=8$, then $\beta \perp \alpha_{1}$ implies that $\beta=-e_{8}-e_{4}$. However, in this case, $(\beta,-\delta)>0$, a contradiction. Thus there is just one possibility for a node $\beta$ that connects to $\alpha_{5}$.

On the other hand, suppose $\beta$ connects to $\alpha_{2}$. Then $\beta$ is orthogonal to $\alpha_{3}, \alpha_{4}$ and $\alpha_{5}$, so the coefficients of $e_{1}, e_{2}, e_{3}$ and $e_{4}$ in $\beta$ are all equal. Furthermore, since $\left(\alpha_{2}, \beta\right)<0$, the nature of the roots in $\Phi$ implies that $\beta=\frac{1}{2}\left(-e_{1}-e_{2}-e_{3}-e_{4}+\cdots\right)$. Since $\beta \perp \alpha_{8}$, the coefficients of $e_{6}$ and $e_{7}$ are equal and hence, since $\beta$ must have an even number of minus signs, we have $\beta=\frac{1}{2}\left(-e_{1}-e_{2}-e_{3}-e_{4}+a e_{5}+b e_{6}+b e_{7}+a e_{8}\right)$, where $a, b= \pm 1$. Of course, $\beta \perp \alpha_{1}$ and this yields $b=1$, so there are two remaining possibilities for $\beta$. Finally, $\left(\alpha_{7}, \beta\right)=\frac{1}{2}(1-a)$ and $(-\delta, \beta)=\frac{1}{2}(-1-a)$ imply that $a=1$ is a solution, but that $a=-1$ is not. Thus there is just one possibility for a node $\beta$ that connects to $\alpha_{2}$.

We conclude from the above that $m_{1}=2$. Since $m_{2}$ is clearly equal to 1 , we have $\operatorname{Ind}\left(P_{\lambda}\right)=m_{1} / m_{2}=2 / 1$, and since $c=4$, Theorem 4.2 implies that the number of composite functions $\kappa \circ \lambda$ is given by

$$
\# \kappa \circ \lambda=\frac{|\mathcal{W}(\Phi)|}{\left|\mathcal{W}\left(\Phi_{\lambda}\right)\right|} \cdot \frac{\phi(c)}{\operatorname{Ind}\left(P_{\lambda}\right)}=\frac{\left(2^{14} \cdot 3^{5} \cdot 5^{2} \cdot 7\right) \cdot 2}{\left(2^{4} \cdot 5 !\right) \cdot 4 ! \cdot 2}=2^{4} \cdot 3^{3} \cdot 5 \cdot 7
$$


Since $P=P_{\lambda}$ for some maximal $\lambda$ if and only if $P$ is a one-step subalgebra of $L$, the work above actually counts the number of such one-step subalgebras that contain a fixed Cartan subalgebra of $L$.

Additional applications of the relationship between $\lambda$ and $P_{\lambda}$ are considered in the next section.

\section{Associated graded Lie Algebras}

If $\lambda: V \rightarrow \mathbb{R}$ is a linear functional, then $\mathcal{F}_{\lambda}=\left\{F_{i} \mid i \in \mathbb{Z}\right\}$ is a bounded filtration of $L$, and it is appropriate to study its associated graded Lie algebra $G_{\lambda}=\oplus \sum_{r \in \mathbb{Z}} F_{r} / F_{r-1}$. Much of the following result holds for arbitrary functionals, but certainly key parts require that $\lambda$ be maximal.

Proposition 5.1. Let $\lambda$ be a maximal functional of order $c$, and let $G_{\lambda}$ denote the associated graded Lie algebra of $\mathcal{F}_{\lambda}=\left\{F_{i} \mid i \in \mathbb{Z}\right\}$. Then

i. $G_{\lambda}=N_{\lambda} \rtimes P_{\lambda}$, where $N_{\lambda}=\operatorname{rad} G_{\lambda}$ is nilpotent of class $<c$.

ii. $N_{\lambda}$ is a $\mathbb{Z} / c \mathbb{Z}$-graded Lie algebra with trivial 0-component and with all remaining components nontrivial.

iii. $N_{\lambda}$ is isomorphic to $L / P_{\lambda}$ as an ad $P_{\lambda}$-module.

$i v$. The nonzero $\mathbb{Z} / c \mathbb{Z}$-components are the irreducible ad $P_{\lambda}$-submodules of $N_{\lambda}$.

Proof. Again, let $\Phi^{\prime}=\Phi \cup\{0\}$ and, for each $1 \leq i \leq c$, define $S_{i} \subseteq L$ to be the sum of those spaces $L_{\alpha}$ with $\alpha \in \Phi^{\prime}$ and $\lambda(\alpha) \in \mathbb{Z}+(i / c)$. Then $L=\oplus \sum_{i=1}^{c} S_{i}$ and, by Proposition 3.1 and Theorem 4.2, each $S_{i}$ is nonzero. In the following, we use an overbar to denote the image of appropriate elements of $L$ in $G_{\lambda}$. Indeed, since each $F_{r}$ is a sum of various root spaces, it is clear that $G_{\lambda}=\sum_{\alpha \in \Phi^{\prime}} \bar{L}_{\alpha}$.

Now let $\alpha, \beta \in \Phi^{\prime}$ with $\lambda(\alpha) \in \mathbb{Z}+(i / c), \lambda(\beta) \in \mathbb{Z}+(j / c)$, and $1 \leq i, j \leq c$. Then there exist suitable integers $r$ and $s$ with $\lambda(\alpha)=r-1+(i / c)$ and $\lambda(\beta)=s-1+(j / c)$, so $\bar{L}_{\alpha} \subseteq F_{r} / F_{r-1}$ has grade $r$ in $G_{\lambda}$ and $\bar{L}_{\beta} \subseteq F_{s} / F_{s-1}$ has grade $s$. If $\alpha+\beta \notin \Phi^{\prime}$, then $\left[L_{\alpha}, L_{\beta}\right]=0$ and hence $\left[\bar{L}_{\alpha}, \bar{L}_{\beta}\right]=0$. On the other hand, if $\alpha+\beta \in \Phi^{\prime}$, then $\left[L_{\alpha}, L_{\beta}\right] \subseteq L_{\alpha+\beta}$ and $r+s-2<\lambda(\alpha+\beta)=\lambda(\alpha)+\lambda(\beta) \leq r+s$. In particular, if $\lambda(\alpha)+\lambda(\beta) \leq r+s-1$, then $L_{\alpha+\beta} \subseteq F_{r+s-1}$ and hence $\left[\bar{L}_{\alpha}, \bar{L}_{\beta}\right]=0$. Finally, if $\lambda(\alpha)+\lambda(\beta)>r+s-1$, then $\bar{L}_{\alpha+\beta}$ has grade $r+s$ and $\left[\bar{L}_{\alpha}, \bar{L}_{\beta}\right]=\overline{\left[L_{\alpha}, L_{\beta}\right]}$. By the latter, we actually mean the stronger formula that for all $x \in L_{\alpha}$ and $y \in L_{\beta}$, we have $[\bar{x}, \bar{y}]=\overline{[x, y]}$.

Since $\lambda(\alpha)=r-1+(i / c)$ and $\lambda(\beta)=s-1+(j / c)$, we have $\lambda(\alpha)+\lambda(\beta)=$ $(r+s-1)+(i / c+j / c-1)$. Thus, if $i+j \leq c$, then $\left[\bar{L}_{\alpha}, \bar{L}_{\beta}\right]=0$, while if $i+j>c$, then $\left[\bar{L}_{\alpha}, \bar{L}_{\beta}\right]=\overline{\left[L_{\alpha}, L_{\beta}\right]} \subseteq \bar{S}_{i+j-c}$. It now follows that $G_{\lambda}=\sum_{\alpha \in \Phi^{\prime}} \bar{L}_{\alpha}=\sum_{i=1}^{c} \bar{S}_{i}$, and that

$$
\left[\bar{S}_{i}, \bar{S}_{j}\right]= \begin{cases}\left.\overline{\left[S_{i}, S_{j}\right.}\right] \subseteq \bar{S}_{i+j-c}, & \text { if } i+j>c \\ 0, & \text { otherwise }\end{cases}
$$

Again, this formula is written with the understanding that it applies elementwise.

We conclude from the above that $\bar{S}_{c}$ is a Lie subalgebra of $G_{\lambda}$ and that $\bar{S}_{c} \cong S_{c}$. But, clearly $S_{c}=P_{\lambda}$, so $\bar{S}_{c} \cong P_{\lambda}$ is a subalgebra of $G_{\lambda}$. In addition, note that $\left[\bar{S}_{i}, \bar{S}_{c}\right]=\overline{\left[S_{i}, S_{c}\right]} \subseteq \bar{S}_{i}$, so that each $\bar{S}_{i}$ is an ad $\bar{S}_{c}$-module isomorphic to $S_{i}$ as an ad $P_{\lambda}$-module. Next, let $S=\sum_{i=1}^{c-1} S_{i}$ and note that $G_{\lambda}=\bar{S} \oplus \bar{S}_{c}$ and that $[\bar{S}, \bar{S}] \subseteq \bar{S}$. Thus, by the above, $N_{\lambda}=\bar{S}$ is an ideal of $G_{\lambda}$ and hence $G_{\lambda} \cong N_{\lambda} \rtimes P_{\lambda}$. Furthermore, if $T_{i}=S_{1}+S_{2}+\cdots+S_{i}$ with $0 \leq i<c$ and $T_{0}=0$, then $\bar{T}_{i} \triangleleft \bar{S}$ and indeed $\left[\bar{S}, \bar{T}_{i}\right] \subseteq \bar{T}_{i-1}$ for $i \geq 1$. Thus, we see that $\bar{S}=N_{\lambda}$ is nilpotent of 
class $<c$ and, since $P_{\lambda}$ is semisimple, it follows that $N_{\lambda}=\operatorname{rad} G_{\lambda}$. Finally, if we write $\mathbb{Z} / c \mathbb{Z}=\{0,1,2, \ldots, c-1\}$, then the above displayed equation implies that $N_{\lambda}=\sum_{i=1}^{c-1} \bar{S}_{i}$ is $\mathbb{Z} / c \mathbb{Z}$-graded with trivial 0-component.

We have proved parts (i) and (ii), and it remains to consider the ad $P_{\lambda}$-module structure of the various components. To this end, we know that each $S_{i}$, with $1 \leq i<c$ is an ad $P_{\lambda}$-module isomorphic to $\bar{S}_{i}$ as an ad $\bar{S}_{c}$-module. Thus $S$ is an ad $P_{\lambda}$-module isomorphic to $\bar{S}=N_{\lambda}$ as an ad $\bar{S}_{c}$-module. But $L=S \oplus P_{\lambda}$, so $S \cong L / P_{\lambda}$ as an ad $P_{\lambda}$-module, and this yields (iii).

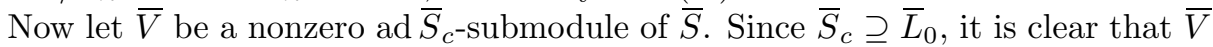
must contain the image $\bar{L}_{\alpha}$ of a root set with $\lambda(\alpha) \in \mathbb{Z}+(i / c)$ for some $1 \leq i<c$. Suppose $\beta$ is any root with $\lambda(\beta) \in \mathbb{Z}+(i / c)$, and use the notation of Lemma 2.1. Then $\lambda\left(\alpha^{\prime}\right), \lambda\left(\beta^{\prime}\right) \in \mathbb{Z}+(i / c)$ and, by Lemma 2.4, either $\alpha^{\prime}=\beta^{\prime}$ or $\alpha^{\prime}-\beta^{\prime} \in \Phi$. In the latter case, we note that $\lambda\left(\alpha^{\prime}-\beta^{\prime}\right) \in \mathbb{Z}$, so $\alpha^{\prime}-\beta^{\prime} \in \Phi_{\lambda}$. Since $\bar{V}$ is an

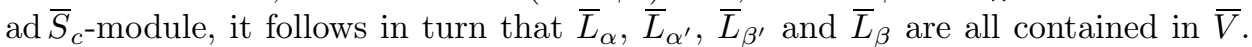
With this, we see that $\bar{V} \supseteq \bar{S}_{i}$, and part (iv) is proved.

We conclude from the above that $\lambda$ has order 1 if and only if $G_{\lambda} \cong L$ and hence if and only if $\mathcal{F}_{\lambda}$ is the filtration associated to a grading of $L$. On the other hand, if $\lambda$ has order 2 , which occurs in most other cases, then $N_{\lambda}$ is commutative and hence $G_{\lambda} \cong\left(L / P_{\lambda}\right) \rtimes P_{\lambda}$. Finally, if $\lambda$ has order $3,4,5$ or 6 , then $N_{\lambda}$ is no longer commutative, so the structure of $G_{\lambda}$ is somewhat more complicated.

\section{REFERENCES}

[BP] Y. Barnea and D. S. Passman, Filtrations in semisimple Lie algebras, I, Trans. AMS, 358 (2006), 1983-2010. MR2197439 (2007a:17013)

[Bo] N. Bourbaki, Lie Groups and Lie Algebras: Chapters 4-6, Springer-Verlag, Berlin, 2002. MR1890629 (2003a:17001)

[D] E. B. Dynkin, Semisimple subalgebras of semisimple Lie algebras, AMS Translations (2) 6 (1957), 111-244.

[H] J. E. Humphreys, Introduction to Lie Algebras and Representation Theory, second printing, Springer-Verlag, New York, 1972. MR0323842 (48:2197)

[K] V. G. Kac, Infinite Dimensional Lie Algebras, Cambridge Univ. Press, Cambridge, 1990. MR1104219 (92k:17038)

Department of Mathematics, Royal Holloway, University of London, Egham, Surrey TW20 0EX, United Kingdom

E-mail address: y.barnea@rhul.ac.uk

Department of Mathematics, University of Wisconsin-Madison, Madison, Wisconsin 53706

E-mail address: passman@math.wisc.edu 